\title{
Preface
}

\section{Update in Neuroanesthesia-An Anesthesiology Clinics Issue Affiliated with SNACC}

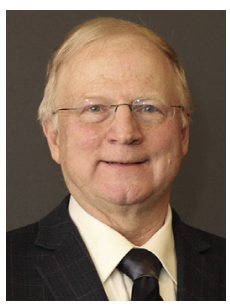

W. Andrew Kofke, MD, MBA

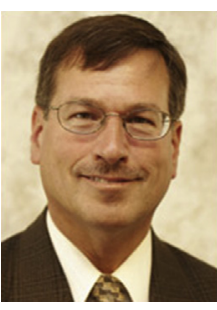

William M. Armstead, PhD

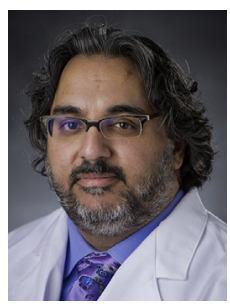

Dhanesh K. Gupta, MD

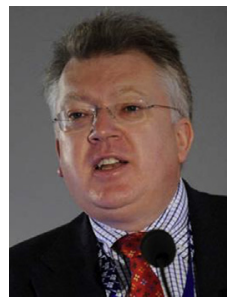

Martin Smith, MBBS, FRCA, FFICM

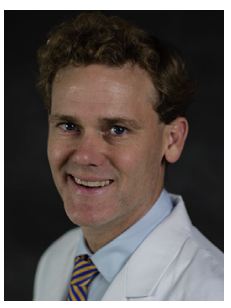

Michael L.

"Luke" James, MD, FAHA, FNCS

Editor

Neuroanesthesiology has a long history as the essence of the practice of anesthesiology, altering the brain to allow surgery and supporting systemic physiology to support the brain. As such, the field has evolved significantly from the era of finger on the pulse and giving urea for intraoperative brain edema. We have dealt with many historic issues, which have included induced hypotension, venous air embolism, circulatory arrest, neuroprotection, measurement and maintenance of cerebral and spinal cord blood flow, neural mechanisms of anesthesia, pain management, and neurotoxicity. Many of these transitions have been well reviewed in two historical pieces on the history of Society for Neuroscience in Anesthesiology and Critical Care (SNACC), one by Albin at 25 years $^{1}$ and one by Kofke at 40 years of SNACC. ${ }^{2}$ This history indicates that the field is serially changing as well, demonstrated by a series of annual reviews by Pasternak and Lanier, ${ }^{3-13}$ such that periodic reviews such as this issue of Anesthesiology Clinics are needed and welcomed.

This issue of Anesthesiology Clinics provides a timely update of several topics relevant to Neuroanesthesiology. Notably, all of the authors are members of the SNACC, and all of the editors are present or past members of the SNACC board of directors. Although the issue has not undergone review for formal endorsement by SNACC, it is nonetheless justifiably considered to be SNACC affiliated with approval of the SNACC board of directors for this designation.

This work is published in collaboration with the Society for Neuroscience in Anesthesiology and Critical Care. 
In this issue, we review recent advances in the broad areas of basic neuroscience, clinical anesthesia practice, intraoperative and critical care monitoring, and aspects of neurocritical care. Within these broad areas, readers will find topics addressing anesthetic action, neuropathophysiology, cerebral blood flow, chronic pain, anesthesia for endovascular management of stroke, multimodality monitoring, brain oxygen monitoring, traumatic brain injury, subarachnoid hemorrhage, and neuromuscular diseases.

Although not a fully comprehensive review of neuroanesthesiology, this issue of Anesthesiology Clinics should provide an authoritative resource on some of the most important aspects of the major areas that comprise and define neuroanesthesiology: neuroscience, clinical anesthesia for neurosurgery and neuroradiology, neuromonitoring, and neurocritical care.

W. Andrew Kofke, MD, MBA Department of Anesthesiology and Critical Care University of Pennsylvania 7 Dulles Building 3400 Spruce Street Philadelphia, PA 19104-4283, USA

William M. Armstead, PhD Department of Anesthesiology and Critical Care University of Pennsylvania 3620 Hamilton Walk, JM3 Philadelphia, PA 19104, USA

Dhanesh K. Gupta, MD Department of Anesthesiology Duke University Medical Center DUMC 3094

Durham, NC 27710, USA

Martin Smith, MBBS, FRCA, FFICM Department of Neuroanaesthesia and Neurocritical Care The National Hospital for Neurology and Neurosurgery University College London Hospitals

Queen Square London WC1N 3BG, UK

Michael L. "Luke" James, MD, FAHA, FNCS Divisions of Neuroanesthesiology and Critical Care Medicine Department of Anesthesiology Division of Neurocritical Care

Department of Neurology

Duke University DUMC-3094

Durham, NC 27710, USA

E-mail addresses: 
dhanesh.gupta@duke.edu (D.K. Gupta)

martin.smith@uclh.nhs.uk (M. Smith)

michael.james@duke.edu (M.L. "Luke" James)

\section{REFERENCES}

1. Albin MS, Albin MS. Celebrating silver: the genesis of a neuroanesthesiology society. NAS->SNANSC->SNACC. Neuroanesthesia Society. Society of Neurosurgical Anesthesia and Neurological Supportive Care. Society of Neurosurgical Anesthesia and Critical Care. J Neurosurg Anesthesiol 1997;9:296-307.

2. Kofke WA. Celebrating ruby: 40 years of NAS $\rightarrow$ SNANSC $\rightarrow$ SNACC $\rightarrow$ SNACC. J Neurosurg Anesthesiol 2012;24:260-80.

3. Pasternak JJ, Lanier WL. Neuroanesthesiology update. J Neurosurg Anesthesiol 2016;28:93-122.

4. Pasternak JJ, Lanier WL. Neuroanesthesiology update. J Neurosurg Anesthesiol 2015;27:87-122.

5. Pasternak JJ, Lanier WL. Neuroanesthesiology update. J Neurosurg Anesthesiol 2014;26:109-54.

6. Pasternak JJ, Lanier WL. Neuroanesthesiology update. J Neurosurg Anesthesiol 2013;25:98-134.

7. Pasternak JJ, Lanier WL. Neuroanesthesiology update. J Neurosurg Anesthesiol 2012;24:85-112.

8. Pasternak JJ, Lanier WL. Neuroanesthesiology update 2010. J Neurosurg Anesthesiol 2011;23:67-99.

9. Pasternak JJ, Lanier WL. Neuroanesthesiology update. J Neurosurg Anesthesiol 2010;22:86-109.

10. Pasternak JJ, Lanier WL. Neuroanesthesiology review-2007. J Neurosurg Anesthesiol 2008;20:78-104.

11. Pasternak JJ, Lanier WL. Neuroanesthesiology review-2006. J Neurosurg Anesthesiol 2007; 19:70-92.

12. Pasternak JJ, Lanier WL. Neuroanesthesiology review-2005. J Neurosurg Anesthesiol 2006;18:93-105.

13. Pasternak JJ, Lanier WL. Neuroanesthesiology review-2004. J Neurosurg Anesthesiol 2005;17:2-8. 